\title{
Diastereoselective Radical Hydrogen Transfer Reactions using N-Heterocyclic Carbene Boranes
}

\author{
Guillaume Tambutet ${ }^{\dagger, \ddagger}$ and Yvan Guindon ${ }^{\dagger, \ddagger} \S$ \\ ${ }^{\dagger}$ Bio-Organic Chemistry Laboratory, Institut de Recherches Cliniques de Montréal (IRCM), Montreal, Quebec \\ H2W 1R7, Canada \\ † Departement de Chimie, Université de Montréal, Montréal, Québec H3C 3J7, Canada \\ $\S$ Department of Chemistry, McGill University, 801 Sherbrooke Street West, Montreal, Quebec H3A 2K6, Canada \\ yvan.guindon@ircm.qc.ca.
}

\section{Contents}

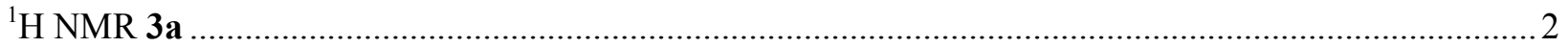

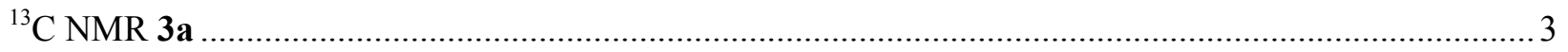

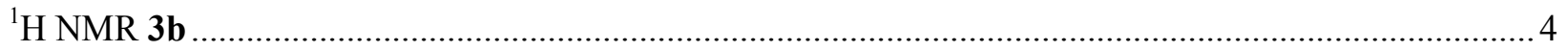

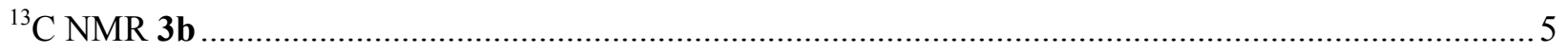

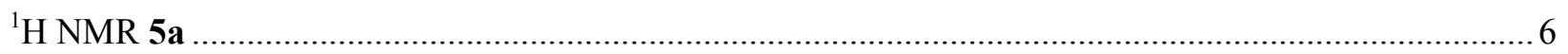

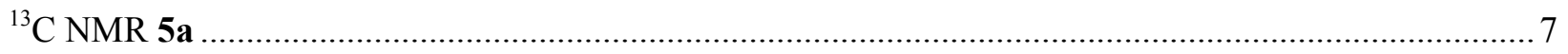

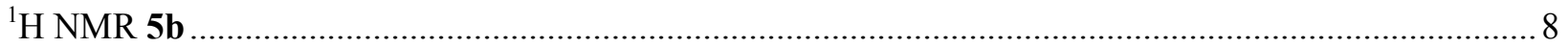

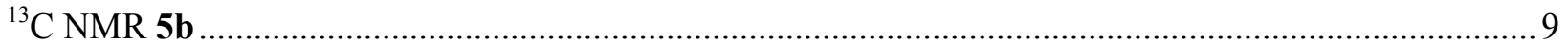


${ }^{1} \mathrm{H}-\mathrm{NMR}\left(500 \mathrm{MHz}, \mathrm{CDCl}_{3}\right.$ ):
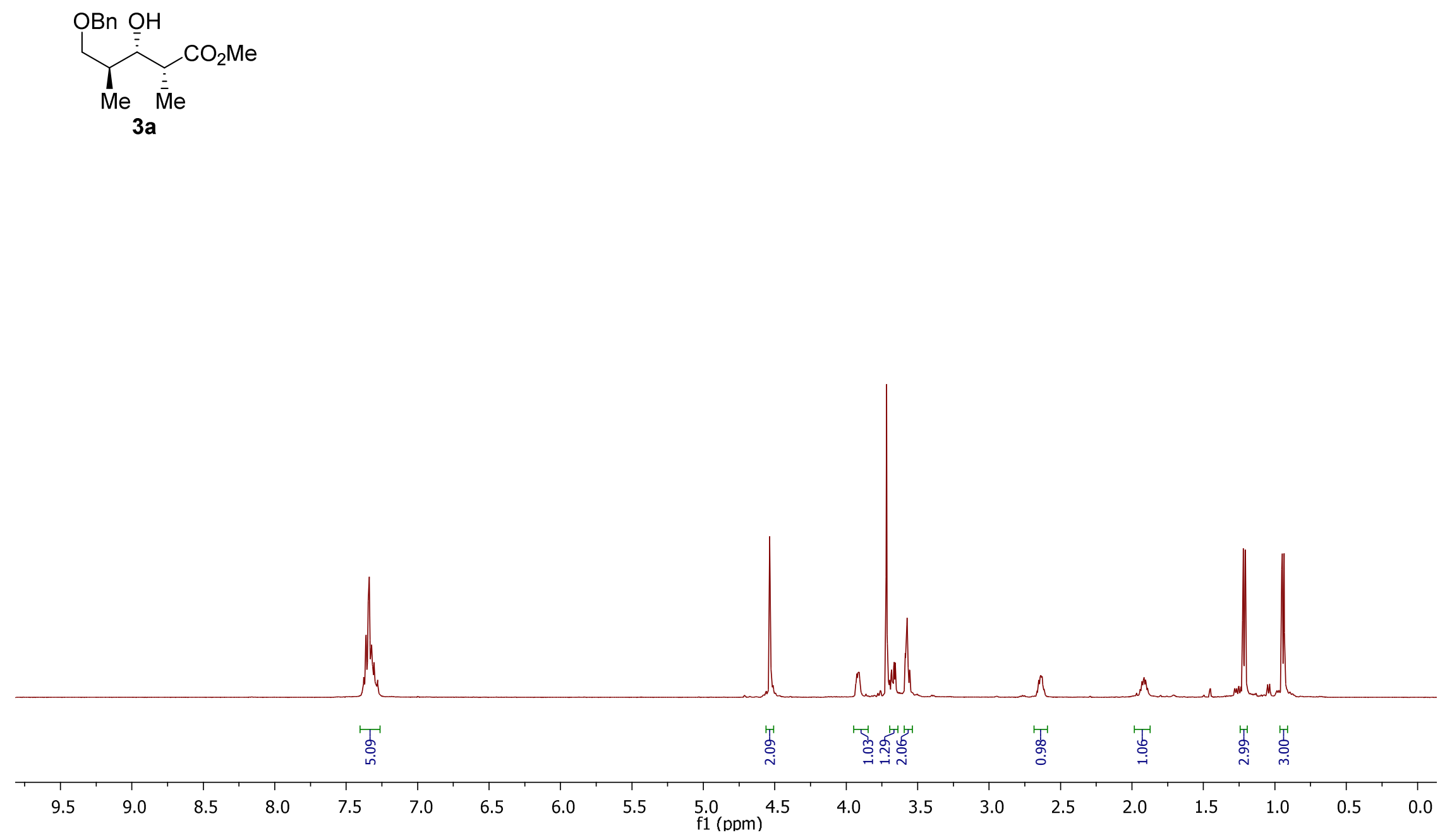
${ }^{13} \mathrm{C}-\mathrm{NMR}\left(125 \mathrm{MHz}, \mathrm{CDCl}_{3}\right)$ :

$$
\underbrace{\mathrm{OBn}}_{\mathrm{Me}} \underbrace{\mathrm{OH}}_{\overline{\overline{\mathrm{M}}}} \mathrm{CO}_{2} \mathrm{Me}
$$$$
\text { 3a }
$$

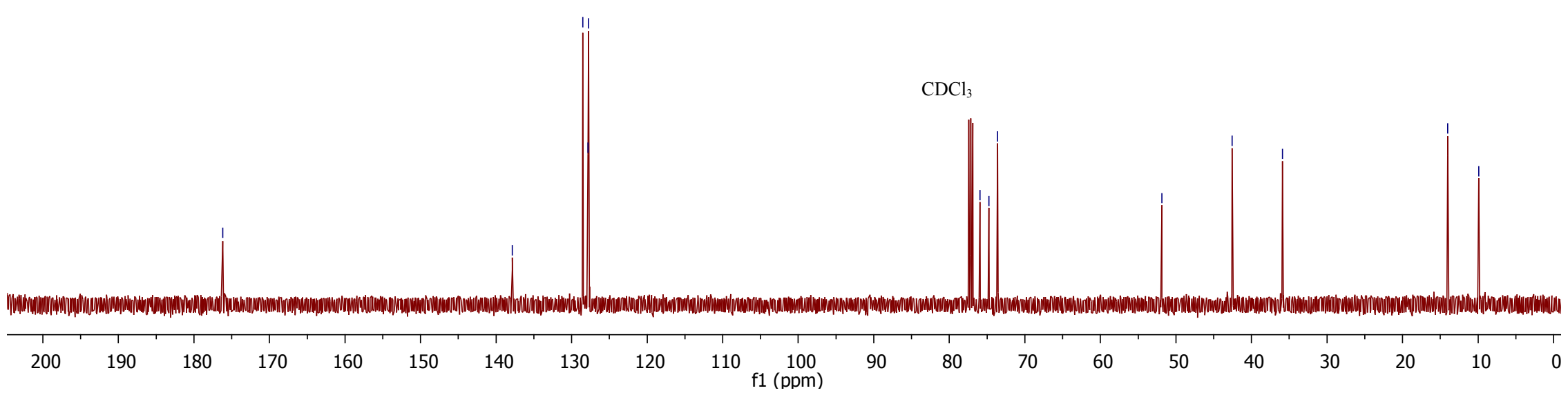


${ }^{1} \mathrm{H}-\mathrm{NMR}\left(500 \mathrm{MHz}, \mathrm{CDCl}_{3}\right.$ ):

$\underbrace{\mathrm{OBn}}_{\mathbf{M e}} \underbrace{\mathrm{OH}}_{\mathbf{3}} \mathrm{CO}_{2} \mathrm{Me}$

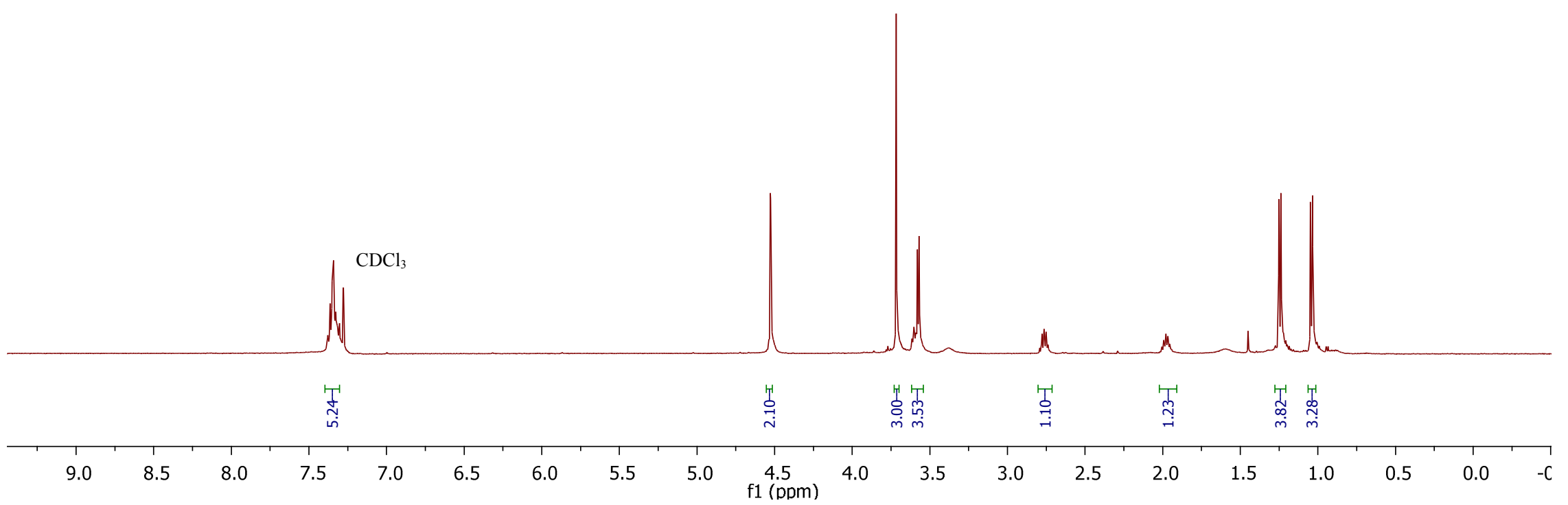




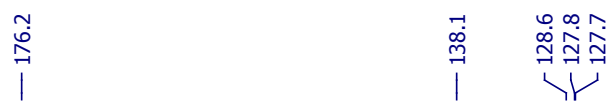

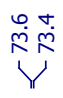

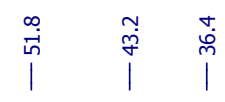

${ }^{13} \mathrm{C}-\mathrm{NMR}\left(125 \mathrm{MHz}, \mathrm{CDCl}_{3}\right)$

$\underbrace{\mathrm{OBn}}_{\mathrm{Me}} \underbrace{\mathrm{OH}}_{\mathbf{M e}} \mathrm{CO}_{2} \mathrm{Me}$

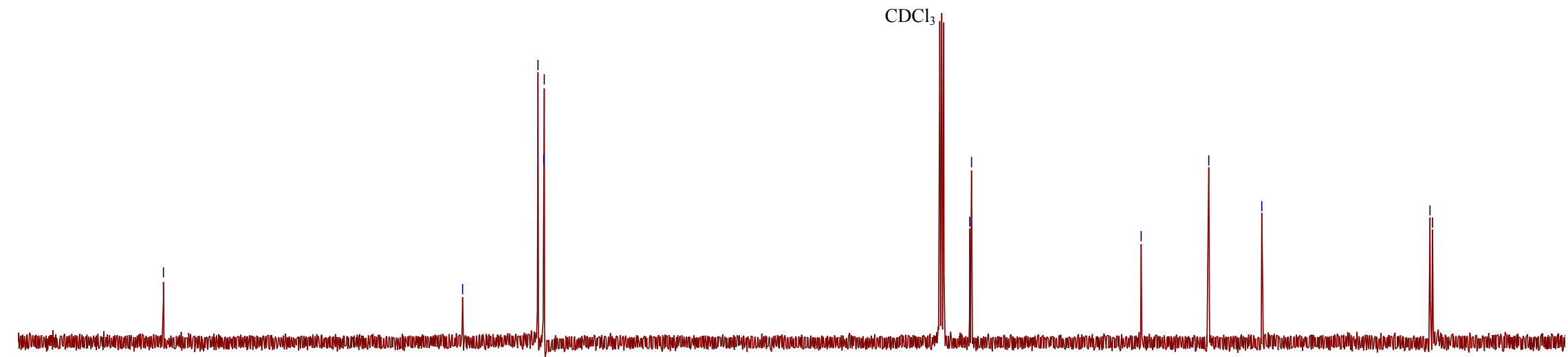


${ }^{1} \mathrm{H}-\mathrm{NMR}\left(500 \mathrm{MHz}, \mathrm{CDCl}_{3}\right)$ :
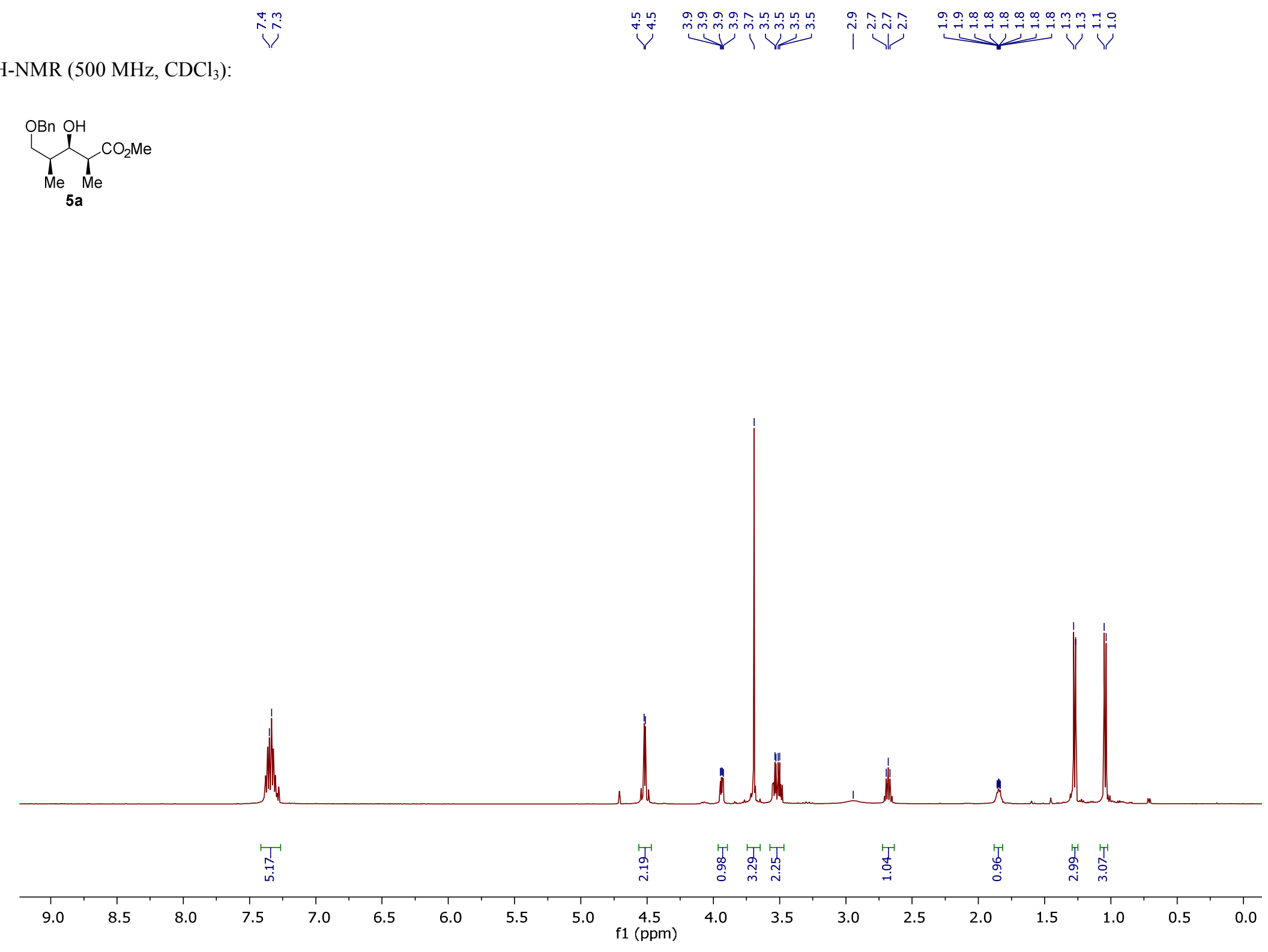
${ }^{13} \mathrm{C}-\mathrm{NMR}\left(125 \mathrm{MHz}, \mathrm{CDCl}_{3}\right)$<smiles></smiles>

$5 a$

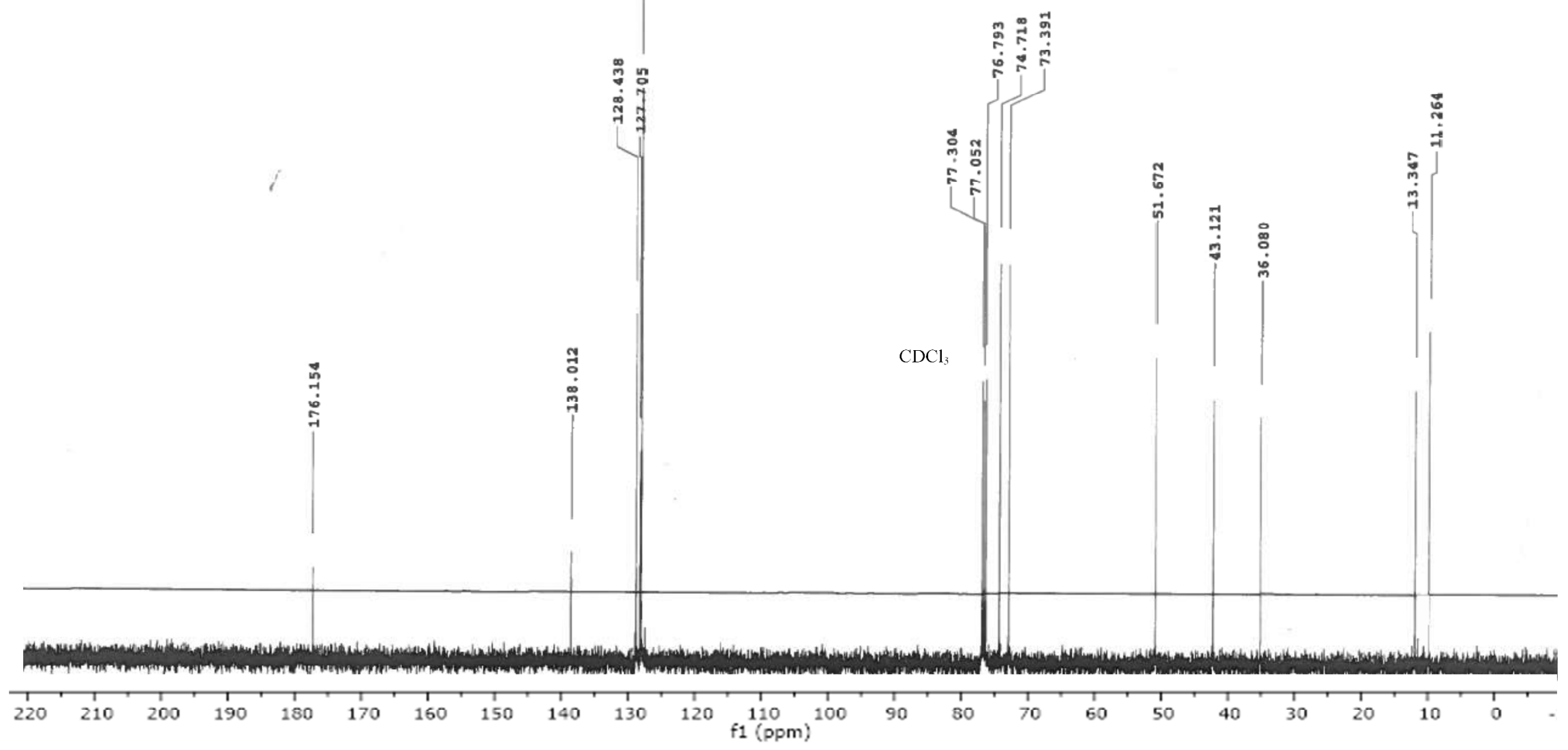


${ }^{1} \mathrm{H}-\mathrm{NMR}$ (500 MHz, $\mathrm{CDCl}_{3}$ ):
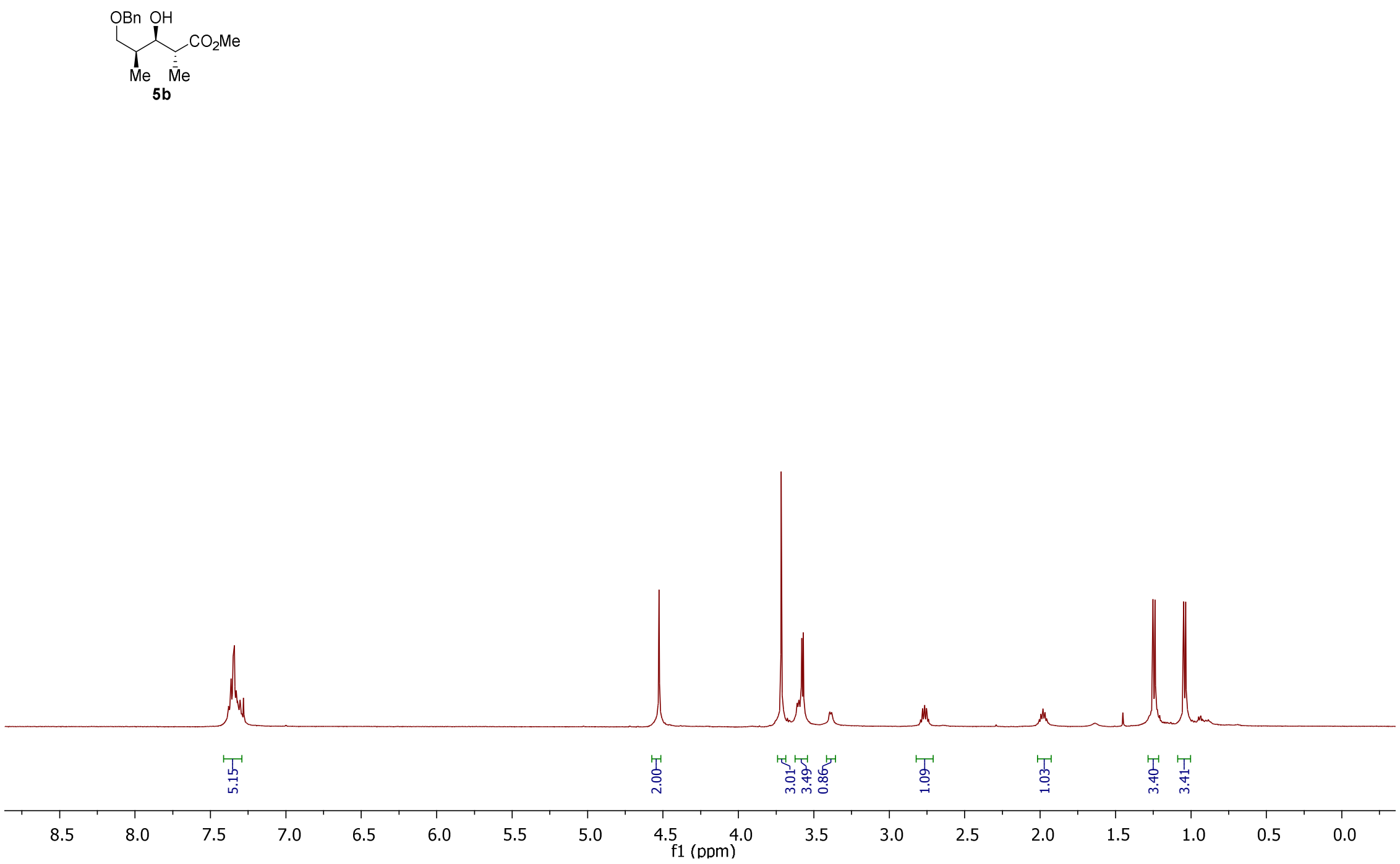
${ }^{13} \mathrm{C}-\mathrm{NMR}\left(125 \mathrm{MHz}, \mathrm{CDCl}_{3}\right)$

$$
\underbrace{\mathrm{Ob}}_{\mathbf{M e}} \underset{\overline{\mathbf{M}}}{\mathrm{O} e}
$$

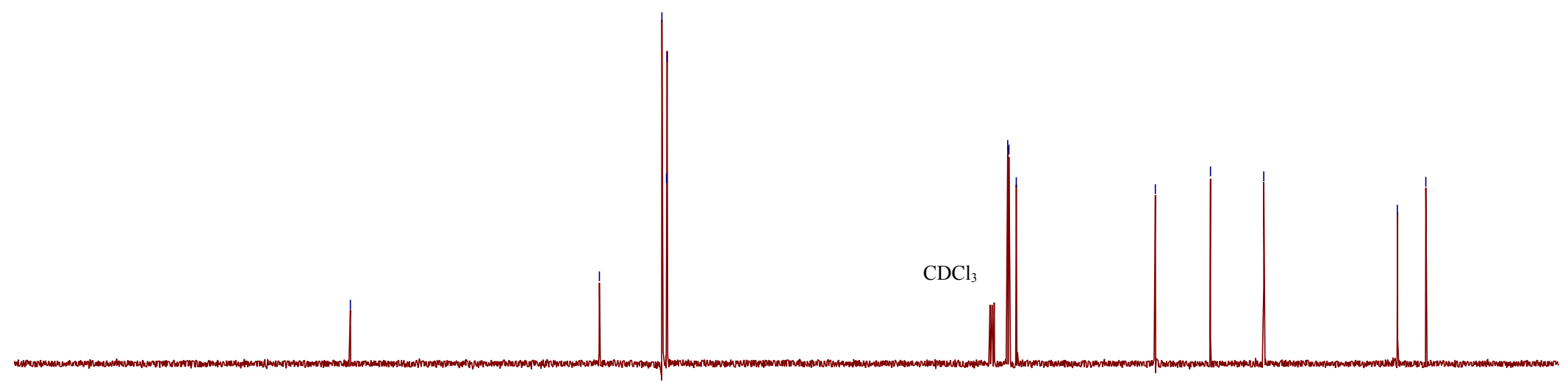

